\title{
Modelling the Emerging COVID-19 Epidemic and Estimating Intervention Effectiveness - Taiwan, China, 2021
}

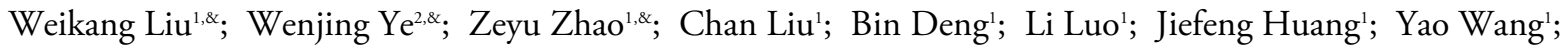 \\ Jia Rui'; Benhua Zhao'; Yanhua Su${ }^{1}$; Shenggen $\mathrm{Wu}^{2}$; Kun $\mathrm{Chen}^{2}$; Jianming $\mathrm{Ou}^{2, *,}$; Tianmu Chen ${ }^{1, *}$
}

\section{ABSTRACT}

Introduction: The coronavirus disease 2019 (COVID-19) pandemic recently affected Taiwan, China. This study aimed to calculate the transmissibility of COVID-19 to predict trends and evaluate the effects of interventions.

Methods: The data of reported COVID-19 cases was collected from April 20 to May 26, 2021, which included daily reported data (Scenario I) and reported data after adjustment (Scenario II). A susceptibleexposed-symptomatic-asymptomatic-recovered model was developed to fit the data. The effective reproductive number $\left(R_{f f}\right)$ was used to estimate the transmissibility of COVID-19.

Results: A total of 4,854 cases were collected for the modelling. In Scenario I, the intervention has already taken some effects from May 17 to May 26 (the $R_{\text {eff }}$ reduced to 2.1 ). When the $R_{\text {eff }}$ was set as 0.1 , the epidemic was projected to end on July 4 , and a total of 1,997 cases and 855 asymptomatic individuals would have been reported. In Scenario II, the interventions were projected as having been effective from May 24 to May 26 (the $R_{a f f}$ reduced to 0.4). When the $R_{f f f}$ was set as 0.1 , the epidemic was projected to end on July 1 , and a total of 1,482 cases and 635 asymptomatic individuals would have been reported.

Conclusion: The epidemic of COVID-19 was projected to end after at least one month, even if the most effective interventions were applied in Taiwan, China. Although there were some positive effects of intervention in Taiwan, China.

\section{INTRODUCTION}

Recently, a coronavirus disease 2019 (COVID-19) epidemic emerged in Taiwan, China, although local authorities announced the epidemic had been controlled since early 2021 (1). An average of 414 daily confirmed cases were reported from May 18 to May 24 in Taiwan, China (2). The epidemic was potentially traced to gatherings of local citizens (3). Furthermore, the epidemic's accelerated spread might also be related to the Alpha variant (B.1.1.7) of COVID-19 (1). Facing this situation, Taiwan, China moved urgently to implement a series of control measures to respond to the epidemic.

Dynamics models have played important roles in analyzing emerging infectious disease such as dengue fever (4). It might also have applications in analyzing COVID-19, including estimating transmissibility, predicting trends, and evaluating the effects of interventions (5). Differing from statistical models, these dynamics models could better explain transmission mechanisms. The dynamics models that are commonly used in COVID-19 are the susceptibleinfectious-recovered and susceptible-exposedinfectious-recovered models (G). However, these models could not capture the influence of asymptomatic infections. Therefore, we developed a susceptible-exposed-symptomatic-asymptomaticrecovered (SEIAR) model that considered transmission via asymptomatic infections. The SEIAR model had been applied in Hunan Province and Jilin Province in China (7-8). In this study, the SEIAR was further applied to analyze COVID-19 transmission in Taiwan, China and to evaluate the effects of some interventions for controlling COVID-19.

\section{METHODS}

\section{Data Collection}

The daily reported data of COVID-19 from April 20 to May 26, 2021 was collected from the website of Taiwan Affairs Office of the State Council (http://www.gwytb.gov.cn). The demographic data was collected from the Chinese National Bureau of Statistics (http://www.stats.gov.cn/). 


\section{Model Development}

The total population was divided into five categories, including susceptible (S), exposed (E), infectious (I), asymptomatic (A), and recovered/removed individuals (R) (Figure 1). The model was based on the following assumptions: 1) susceptible individuals could be infected via contact with symptomatic and asymptomatic individuals, and the transmission rate was defined as $\beta ; 2$ ) the incubation period of symptomatic individuals and the latent period of asymptomatic individuals were $1 / \omega$ and $1 / \omega^{\prime}$, respectively; the proportion of asymptomatic infection was defined as $p$ (where $0 \leq p$ $\leq 1$ ); exposed individuals would become asymptomatic individuals at a rate of $p \omega E$ or become infectious individuals at a rate of $(1-p) \omega^{\prime} E$; and 3$)$ infectious individuals and asymptomatic individuals would become recovered individuals $(R)$ after an infectious period of $1 / \gamma$ and $1 / \gamma^{\prime}$, respectively. The infectious individuals died due to illness at a rate of $f$.

The equations for SEIAR model were as follows:

$$
\begin{aligned}
& \frac{d S}{d t}=-\beta S(I+\kappa A) / N \\
& \frac{d E}{d t}=\beta S(I+\kappa A)-p \omega E-(1-p) \omega^{\prime} E \\
& \frac{d I}{d t}=p \omega E-\gamma I-f I \\
& \frac{d A}{d t}=(1-p) \omega^{\prime} E-\gamma^{\prime} A \\
& \frac{d R}{d t}=\gamma I+\gamma^{\prime} A
\end{aligned}
$$

In this model, the definitions of the parameters were sourced from previous studies (7-8). Combined with previous studies $(6-7)$, the value of parameter $\kappa$ was set as $0.7, p$ was set as $0.3, \omega$ was set as $0.3333, \omega$ ' was set as $0.2, \gamma$ was set as 0.2 , and $\gamma^{\prime}$ was set as 0.1 . According to extracted data, the total population was set at $23,561,236$, and the case-fatality rate $f$ was set as 0.005 .

Because the basic reproductive number $\left(R_{0}\right)$ was difficult to quantify when coupled with the interventions, we used an effective reproductive

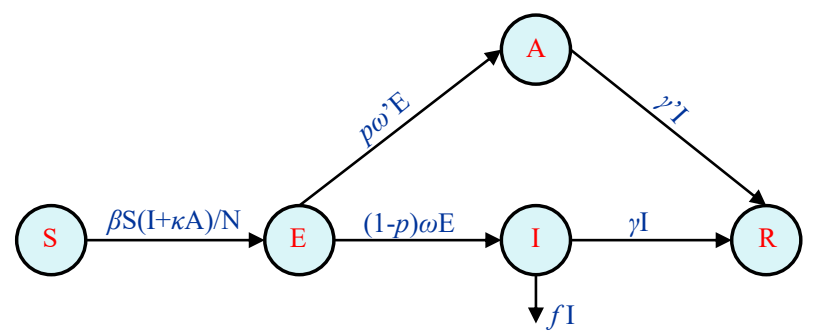

FIGURE 1. The framework of susceptible-exposedsymptomatic-asymptomatic-recovered model. number $\left(R_{\text {eff }}\right)$ to estimate COVID-19 transmissibility. The equation was as follows:

$$
R_{e f f}=\beta\left(\frac{1-p}{f+\gamma}+\frac{\kappa p}{\gamma^{\prime}}\right)
$$

The effectiveness of the interventions was evaluated by examining the decreasing value of $R_{\text {eff }}$. In Jinlin and Zhejiang Province, $R_{\text {eff }}$ dropped below one after the implementation of strict interventions from the national government (8-9). Therefore, the interventions in Taiwan, China could also decrease the value of $R_{\text {eff. }}$.

\section{Statistics Analysis}

The calibration between reported data and the SEIAR model was performed using the least squares method. The coefficient of determination $\left(R^{2}\right)$ was used to evaluate the goodness of fit. We calibrated the data according to the increasing and decreasing trends of reported data. Two scenarios were used to model the data: Scenario I used daily reported data, and Scenario II used daily reported data after correction. The correction was implemented by adjusting daily totals to account for missed cases or delays in reporting or diagnosis.

\section{RESULTS}

A total of 4,854 cases were reported in Taiwan, China from April 20 to May 26, 2021. In addition, over 100 cases were reported daily after May 15 (Figure 2).

In Scenario I (Figure 2A), the model appeared to have a relatively good fit with the data $\left(R^{2}=0.951\right.$, $P<0.001)$. The results showed a total of 4,948 cases and 2,120 asymptomatic individuals before May 26, and the $R_{\text {eff }}$ of the 3 segments were 4.5 (April 20 to May 15), 5.6 (May 15 to May 17), and 2.1 (May 17 to May 26), respectively. The interventions already showed some effects from May 17 to May 26 as the $R_{\text {eff }}$ reduced to 2.1. Based on the above situation, the government was assumed to impose more interventions after May 26 and simulate the sub-scenarios of interventions as follows: 1) if $R_{\text {eff }}=1$, the number of daily reported cases would slowly decrease; a total of 3.2 million cases would be reported from May 26 to August 29, and the epidemic would be not over; 2) if $R_{\text {eff }}=0.5$, the number of daily reported cases would decrease, and the epidemic would end on August 29 and report a total of 0.5 million cases from May 26 to August 29; and 3) if $R_{\text {eff }}=0.1$, the number of daily 


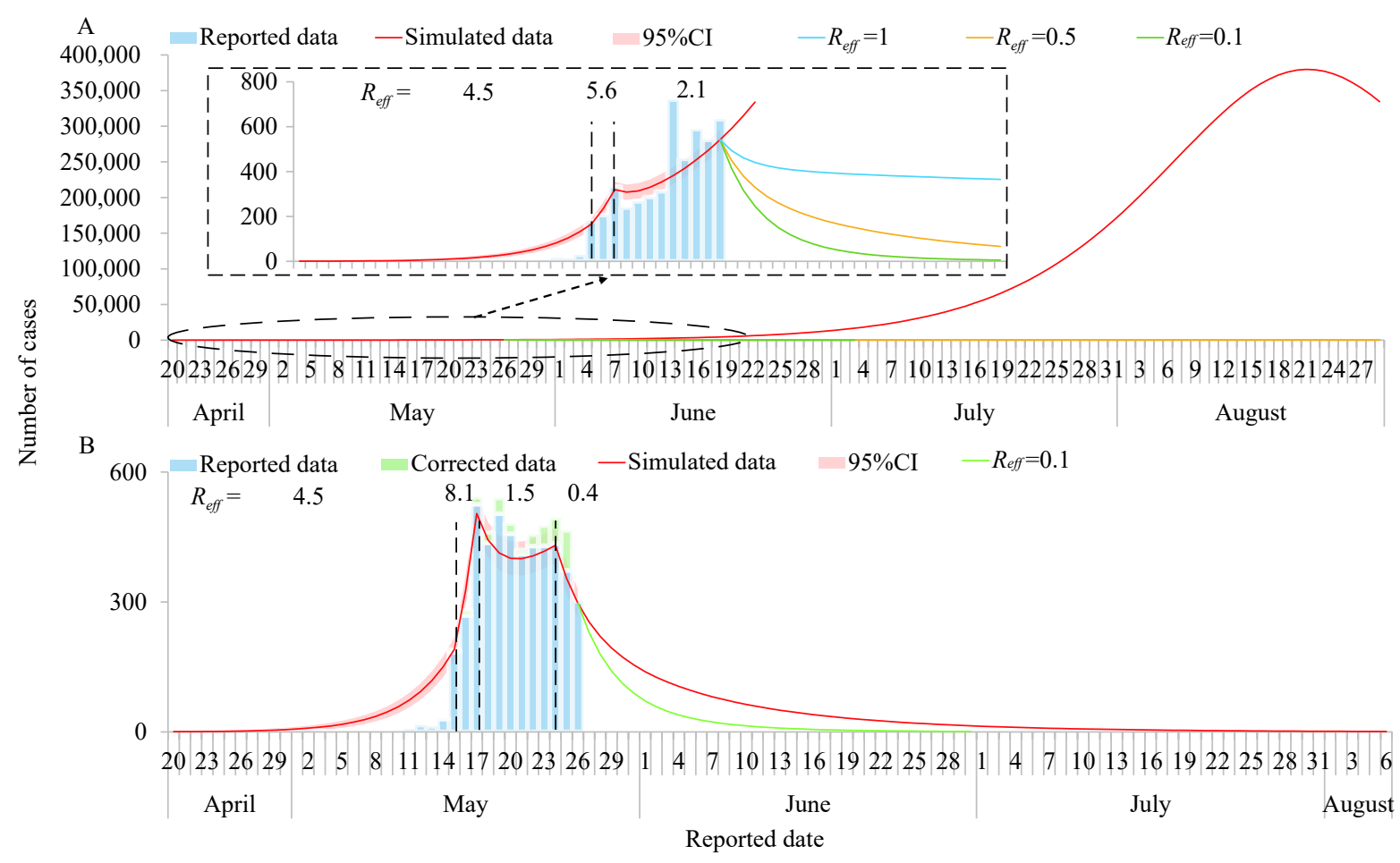

FIGURE 2. The simulation results of epidemic trends based on the different value of $R_{\text {eff }}$ in Taiwan, China, 2021, according to two types of report data. (A) Scenario I; (B) Scenario II.

Note: In scenario I, the daily reported data was directly simulated in the model, and the curve was divided into 3 segments (from April 20 to May 15, from May 15 to May 17, from May 17 to May 26) based on the heterogeneity of the epidemic trends and the best goodness-of-fit of the model. We modeled the data according to trends from May 17 to May 26 and estimated the effects of interventions after May 26 by setting $R_{\text {eff }}$ to $1,0.5$, and 0.1 . In scenario II, the daily reported data after adjustment was simulated in the model, the calibration of curve was divided into four segments (from April 20 to May 15, from May 15 to May 17, from May 17 to May 24, from May 24 to May 26) according to the heterogeneity of the epidemic trends and the best goodness-of-fit of the model. We predicted the data according to the trend from May 24 to May 26 , and performed intervention after May 26 when setting $R_{\text {eff }}$ to 0.1 .

reported cases would quickly decrease, a total of 1,997 cases and 855 asymptomatic individuals would be reported from May 26 to July 4, and the epidemic would end on July 4.

In Scenario II (Figure 2B), the model fitted the data $\left(R^{2}=0.968, P<0.001\right)$ from April 20 to May 26 after regression correction. There were 4,847 confirmed cases after regression correction. A total of 5,293 cases and 2,268 asymptomatic individuals were simulated in our model before May 26, and the $R_{\text {eff }}$ of 4 segments were 4.5 (April 20 to May 15), 8.1 (May 15 to May 17), 1.5 (May 17 to May 24), and 0.4 (May 24 to May 26), respectively. The interventions had already shown effectiveness from May 24 to May 26, with the $R_{\text {eff }}$ decreasing to 0.4 . A total of 2,758 cases and 1,182 asymptomatic individuals would be reported from May 26 to August 7, and the epidemic would end on August 7. Furthermore, if the government imposed more interventions after May 26 and a sub-scenario of $R_{\text {eff }}=0.1$ was simulated, the epidemic would end on
July 1 with a total of 1,482 cases and 635 asymptomatic individuals from May 26 to July 1.

\section{DISCUSSION}

In this study, the SEIAR model was used to calculate the transmissibility of COVID-19 to predict the development trends and evaluate the effectiveness of interventions in Taiwan, China. The results showed that the $R_{\text {eff }}$ of the first segment was 4.5, and the second segment was 5.6. Further interventions are suggested to be implemented to control the epidemic.

The transmissibility in the first stage of the epidemic (April 20 to May 15) in Taiwan, China was 4.4 or 4.5, which suggested that one of the infectious or asymptomatic individuals could infect 4.4 to 4.5 susceptible individuals after contact if no interventions were adopted. A study reported that the alpha variant of COVID-19 (B.1.1.7) had a $43 \%$ to $90 \%$ higher reproductive number than preexisting variants (10). 
Therefore, imported cases might have had the alpha variant and led to the epidemic in Taiwan, China (1). Furthermore, large gatherings were also potential factors accelerating the epidemic. Increased contact frequency also heightened the risk of transmission for COVID-19.

Several interventions have already been implemented for the epidemic. For example, the local authorities announced an upgraded alert for epidemic prevention and control to the third level in Taipei City and Xinbei City on May 15. There are 4 levels of alert, and a larger number means stricter measures. Taiwan, China have also suspended entry of foreigners and stopped passengers from transferring on flights since May 19. A reduction in the value of $R_{\text {eff }}$ had already been observed, but the local authorities should further strengthen interventions to accelerate the end of the COVID-19 epidemic, which was projected to end after at least one additional month with effective interventions $\left(R_{\text {eff }}=0.1\right)$. Therefore, the following measures are suggested for implementation or strengthening: 1) strictly tracing close contacts and enhancing testing of symptomatic and asymptomatic individuals; 2) treat cases according to severity; 3) employing community management measures and maintaining social distancing, and; 4) administering vaccinations as soon as possible and improving the vaccine coverage (11).

This study was subject to at least 3 limitations. First, the parameters of the model were not calculated from first-hand data in Taiwan, China, which might lead to some uncertainty of the simulated results. Second, we did not add the effect of vaccination into the prediction of the model. Third, the effectiveness of each intervention could not be quantified in this model due to limits in the data.

Conflicts of interest: No conflicts of interest were reported.

Funding: Bill \& Melinda Gates Foundation (INV005834), the Science and Technology Program of Fujian Province (No: 2020Y0002), and Provincial Natural Science Foundation of Fujian Province (No: 2020J01094). doi: $10.46234 / \mathrm{ccdcw} 2021.177$

\# Corresponding authors: Jianming Ou, ojmfj@vip.sina.com; Tianmu Chen, chentianmu@xmu.edu.cn.

\footnotetext{
${ }^{1}$ State Key Laboratory of Molecular Vaccinology and Molecular Diagnostics, School of Public Health, Xiamen University, Xiamen, Fujian, China; ${ }^{2}$ Fujian Provincial Center for Disease Control and Prevention, Fuzhou, Fujian, China.

\& Joint first authors.
}

Submitted: May 29, 2021; Accepted: August 05, 2021

\section{REFERENCES}

1. BBC News. Covid-19: what went wrong in Singapore and Taiwan? 2021. https://weibo.com $/ 5225406045 / \mathrm{Kgon} 8 \mathrm{uUgB}$ ? $\bmod =$ weibotime\& type=comment\#_rnd1628752480978. [2021-5-26]. (In Chinese).

2. World Health Organization. WHO coronavirus (COVID-19) dashboard. 2021. https://covid19.who.int/. [2021-5-26].

3. Channel News Asia. What is the connection between wanhua teahouse, Yilan recreation ground, and Lions club. https://www.cna.com.tw/ news/firstnews/202105125016.aspx. [2021-5-12]. (In Chinese).

4. Liu XC, Zhang M, Cheng Q, Zhang YT, Ye GQ, Huang XQ, et al. Dengue fever transmission between a construction site and its surrounding communities in China. Parasit Vectors 2021;14(1):22. http://dx.doi.org/10.1186/s13071-020-04463-x.

5. Zhao QL, Wang Y, Yang M, Li MN, Zhao ZY, Lu XR, et al. Evaluating the effectiveness of measures to control the novel coronavirus disease 2019 in Jilin Province, China. BMC Infect Dis 2021;21(1):245. http://dx.doi.org/10.1186/s12879-021-05936-9.

6. Chen TM, Rui J, Wang QP, Zhao ZY, Cui JA, Yin L. A mathematical model for simulating the phase-based transmissibility of a novel coronavirus. Infect Dis Poverty 2020;9(1):24. http://dx.doi.org/ 10.1186/s40249-020-00640-3.

7. Zhao ZY, Zhu YZ, Xu JW, Hu SX, Hu QQ, Lei Z, et al. A fivecompartment model of age-specific transmissibility of SARS-CoV-2. Infect Dis Poverty 2020;9:117. http://dx.doi.org/10.1186/s40249-02000735-x.

8. Zhao QL, Yang M, Wang Y, Yao LS, Qiao JG, Cheng ZY, et al. Effectiveness of interventions to control transmission of reemergent cases of COVID-19 - Jilin province, China. China CDC Wkly 2020;2(34):651 - 4. http://dx.doi.org/10.46234/ccdcw2020.181.

9. Lao XY, Luo L, Lei Z, Fang T, Chen Y, Liu YH, et al. The epidemiological characteristics and effectiveness of countermeasures to contain coronavirus disease 2019 in Ningbo City, Zhejiang Province, China. Sci Rep 2021;11:9545. http://dx.doi.org/10.1038/s41598-02188473-4.

10. Davies NG, Abbott S, Barnard RC, Jarvis CI, Kucharski AJ, Munday JD, et al. Estimated transmissibility and impact of SARS-CoV-2 lineage B.1.1.7 in England. Science 2021;372(6538):eabg3055.http://dx.doi.org/ 10.1126/science.abg3055.

11. Lai SJ, Ruktanonchai NW, Zhou LC, Prosper O, Luo W, Floyd JR, et al. Effect of non-pharmaceutical interventions to contain COVID-19 in China. Nature 2020;585(7825):410 - 3. http://dx.doi.org/10.1038/ s41586-020-2293-x. 